\title{
Quantification of the mixing effect in silicon-manganese thin films by swift heavy ion irradiation
}

\author{
K Diva ${ }^{1}$, B R Chakraborty ${ }^{1}$, R S Chauhan ${ }^{2}$, J C Pivin $^{3}$ and D K Avasthi ${ }^{4}$ \\ ${ }^{1}$ National Physical Laboratory (CSIR), New Delhi - 110012, India \\ ${ }^{2}$ Department of Physics, RBS College, Agra - 282002, India \\ ${ }^{3}$ CSNSM-IN2P3, Batiment 108, 91405 Orsay Campus, France \\ ${ }^{4}$ Inter-University Accelerator Centre, New Delhi - 110067, India \\ E-mail: divashanker@gmail.com
}

Received 30 May 2008, in final form 24 July 2008

Published 29 August 2008

Online at stacks.iop.org/JPhysD/41/185305

\begin{abstract}
Swift heavy ion induced mixing is reported in a-Si/Mn/a-Si thin films on a silicon wafer, when irradiated by $120 \mathrm{MeV} \mathrm{Au}$ ions at three different fluences of $1 \times 10^{13}, 3 \times 10^{13}$ and $1 \times 10^{14}$ ions $\mathrm{cm}^{-2}$. The samples were characterized before (pristine) and after irradiation using secondary ion mass spectroscopy (SIMS) and Rutherford backscattering spectroscopy (RBS). Atomic force microscopy of the samples was used to determine the surface roughness contribution to RBS and SIMS profiles. Depth profiles showed distinct changes in the interface region and it was observed that interface mixing increased linearly with the increase in the ion fluence. The mixing rate was estimated to be $\sim 1000 \mathrm{~nm}^{4}$. The mixing effect is explained in the framework of the thermal spike model. The track radius and duration of the transient melt phase have been theoretically calculated for this system to estimate the diffusivity during the transient melt stage at the interface.
\end{abstract}

(Some figures in this article are in colour only in the electronic version)

\section{Introduction}

Swift heavy ion (SHI) irradiation is an efficient means to modify the composition and structure of thin films and interfaces [1]. Amid different techniques to modify thin films, SHI irradiations find their effectiveness in terms of their spatial selectivity, precise control and low temperature process. Since the early 1990s SHI-induced mixing of thin films has been studied in various types of systems [2-6]. Srivastava et al have studied in detail the mechanism of SHI-induced mixing [3], which is quite helpful in understanding the mixing behaviour at high energies $\left(\geqslant 1 \mathrm{MeV} a m u^{-1}\right)$. The mixing phenomenon in the electronic regime of slowing down has been primarily explained by either Coulomb explosions or thermal spikes $[3,7,8]$. Their relevance has been checked for different types of systems [9-12]. For metal/semiconductor and metal/metal systems the thermal spike model is more appropriate $[11,12]$.

In continuation of our previous work [11, 13] on amorphous $\mathrm{Si} / \mathrm{TM}$ (transition metal)/Si systems (a-Si/V/a-Si,
a-Si/Fe/a-Si and a-Si/Co/a-Si) we consider here the mixing of another transition metal, Mn, with amorphous $\mathrm{Si}$ (a-Si). $\mathrm{Mn}$ with a-Si is chosen as hardly any detailed study has been done on this system in the high-energy regime. Though Mn is reported to be ' $S_{\mathrm{e}}$-sensitive' (materials showing defect production due to electronic energy loss [8]) still we do not have an apparent picture of its behavioural changes due to electronic energy loss $\left(S_{\mathrm{e}}\right)$. Even the information about its $S_{\mathrm{e}}$-threshold value is not available. Therefore, this system has been selected for SHI-induced mixing studies. This work is an attempt to quantify SHI-induced interface mixing in terms of the mixing rate and thereby to understand this phenomenon in the framework of the thermal spike model. The track radius and duration of the transient melt phase have been theoretically calculated for this system to estimate the diffusivity during the transient molten state at the interface.

Although pure metals have good electrical conductivities, they are not suitable for very large scale integration, ultra large scale integration (VLSI/ULSI) applications. In contrast, metal 
silicides find wide applications in the fabrication of microelectronic devices $[14,15]$, because they often exhibit metallic conduction and they have attracted considerable attention in the context of metallization applications [16]. They also show high temperature stability. Mn silicides are especially interesting for thermoelectric and opto-electronic devices [15]. Metallization, a process to form metal-like layers in integrated circuit (IC) structures, has a high impact on the IC performance. One of the methods of producing metal silicides is ion beam mixing. Despite the fact that a substantial amount of work is done on ion beam induced metal silicides $[17,18]$, it is mostly in the low energy region (a few tens of $\mathrm{keV} \mathrm{amu}^{-1}$ ) where elastic collisions (nuclear energy loss, $S_{\mathrm{n}}$ ) are predominant. An interesting aspect of SHI irradiation is that through SHI the formation of a silicide can take place in a narrow tube and in the crystalline form whereas by irradiation in the ballistic regime we get amorphous silicides.

In this work, the mixing rate $(k)$ for $\mathrm{Si} / \mathrm{Mn} / \mathrm{Si}$ super-imposed layers was determined with the help of two complementary techniques Rutherford backscattering spectroscopy (RBS) and secondary ion mass spectroscopy (SIMS) $[19,20]$ and thereby the diffusion coefficient has been estimated for this system to verify the hypothesis of melt phase diffusion across the interface using the thermal spike model. Atomic force microscopy (AFM) of the samples was performed to verify if there is any surface roughness contribution to the depth profiles of RBS.

\section{Experimental}

Thin films of a-Si/Mn/a-Si were deposited on silicon $\left\langle\begin{array}{lll}1 & 0 & 0\end{array}\right)$ substrates by electron beam evaporation in the UHV deposition system at a base pressure of $\sim 1.5 \times 10^{-8}$ Torr. This was a cryo-pumped system equipped with a four-pocket electron gun. First the Si wafers were sequentially cleaned using trichloroethylene, acetone and methanol. These were then treated with $50 \%$ HF to remove the surface oxide layer. Finally, these were washed with de-ionized water before being transferred to the evaporator. In order to avoid the interference of the native silicon oxide layer during ion beam mixing, a layer of a-Si of thickness $\sim 50 \mathrm{~nm}$ was deposited on the substrate. Mn of $\sim 50 \mathrm{~nm}$ was deposited on this a-Si layer without exposing the deposited Si to atmosphere. Finally an amorphous Si protective layer $(\sim 50 \mathrm{~nm})$ was deposited to avoid the oxidation of the metal layer. The deposition of all the three layers $\mathrm{Si} / \mathrm{Mn} / \mathrm{Si}$ was done without breaking the vacuum to minimize impurities at the interface.

This system has been irradiated with $120 \mathrm{MeV}$ Au ions in a fluence range of $1 \times 10^{13}-1 \times 10^{14}$ ions cm$^{-2}$. The interface region was then analysed by two complementary techniques RBS and SIMS. The samples were mounted on a heavy copper ladder with a good thermal contact using a thermally conducting adhesive, ensuring negligible increase in the sample temperature during irradiation. The samples were irradiated uniformly at room temperature over an area of $1 \mathrm{~cm} \times$ $1 \mathrm{~cm}$ by scanning the ion beam using an electromagnet scanner. The pressure in the irradiation chamber was $\sim 10^{-6}$ Torr. The irradiation fluence was estimated by measuring the ladder

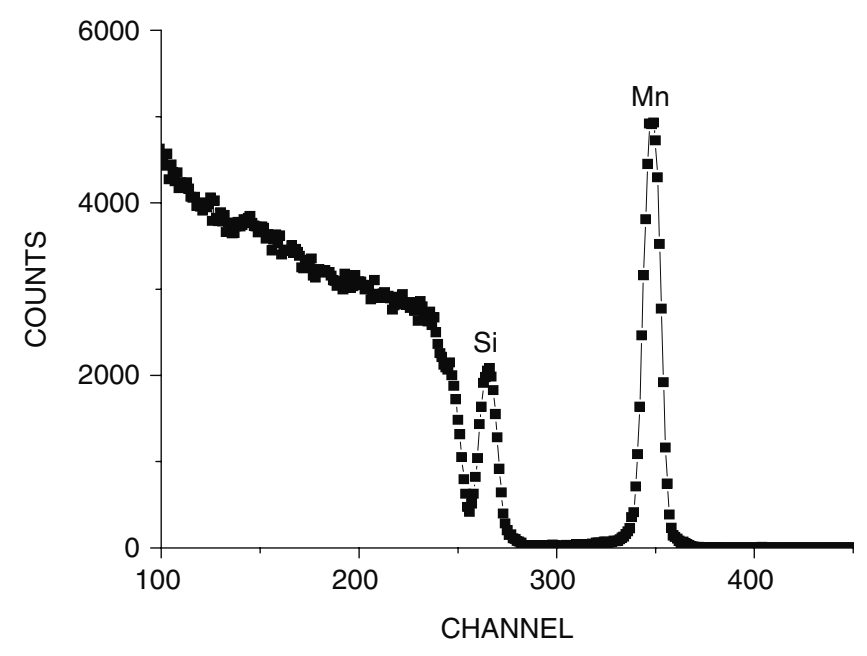

Figure 1. RBS spectrum of the pristine a-Si/Mn/a-Si.

current under secondary-electron-suppressed geometry and taking the scanned ion beam area.

The SHI-induced interface mixing was studied by characterizing the depth profiles of composition before and after irradiation using SIMS and RBS. The RBS experiments were performed on both the un-irradiated and irradiated samples using a $1.0 \mathrm{MeV} \mathrm{He}$ beam at normal incidence, with a collection of the scattered ions at an angle of $165^{\circ}$. A quadrupole type spectrometer was used, for studying the depth profiles by SIMS. The depth profiling of the $\mathrm{Si} / \mathrm{Mn} / \mathrm{Si}$ samples was carried out with oxygen primary ions having $9 \mathrm{keV}$ energy with a beam current of $250 \mathrm{nA}$ and the +ve secondary ions were collected for analysis. The incident angle was taken to be $45^{\circ}$ normal to the surface. The optimization of the different experimental conditions during sputtering was done, keeping in mind of having the best depth resolution. The SIMS parameters remained the same for all the samples of this system irradiated at different fluences.

The AFM experiments were done at IUAC, using a Nanoscope IIIa scanning probe microscope in the tapping AFM mode on both the pristine and the sample irradiated with the highest fluence $\left(1 \times 10^{14}\right.$ ions $\left.\mathrm{cm}^{-2}\right)$. The scanned area was of $1 \mu \mathrm{m} \times 1 \mu \mathrm{m}$ in both the cases.

Energy loss values for $120 \mathrm{MeV} \mathrm{Au}$ ions as calculated from the simulation programme SRIM for this case show that the $S_{\mathrm{e}}$ values are much higher than the $S_{\mathrm{n}}$ values. For $\mathrm{Si}, S_{\mathrm{e}}$ is $14 \mathrm{keV} \mathrm{nm}^{-1}$ and $S_{\mathrm{n}}$ is $0.21 \mathrm{keV} \mathrm{nm}^{-1}$ and for $\mathrm{Mn}$, $S_{\mathrm{e}}$ is $51 \mathrm{keV} \mathrm{nm}^{-1}$ and $S_{\mathrm{n}}$ is $0.38 \mathrm{keV} \mathrm{nm}^{-1}$. Thus, it is observed that the energy loss process by electronic excitation is dominant. Therefore, the observed defects at the interface can be envisaged due to the electronic energy loss deposition. From the existing literature in low energy ion beam mixing, fluences of $10^{15}$ ions $\mathrm{cm}^{-2}$ or higher are used to obtain significant measurable mixing, whereas in the present case the maximum fluence used is only $10^{14}$ ions $\mathrm{cm}^{-2}$.

\section{Results and discussion}

Figure 1 shows the RBS spectrum of the pristine (un-irradiated) sample. It was simulated using the RUMP simulation code [21] 

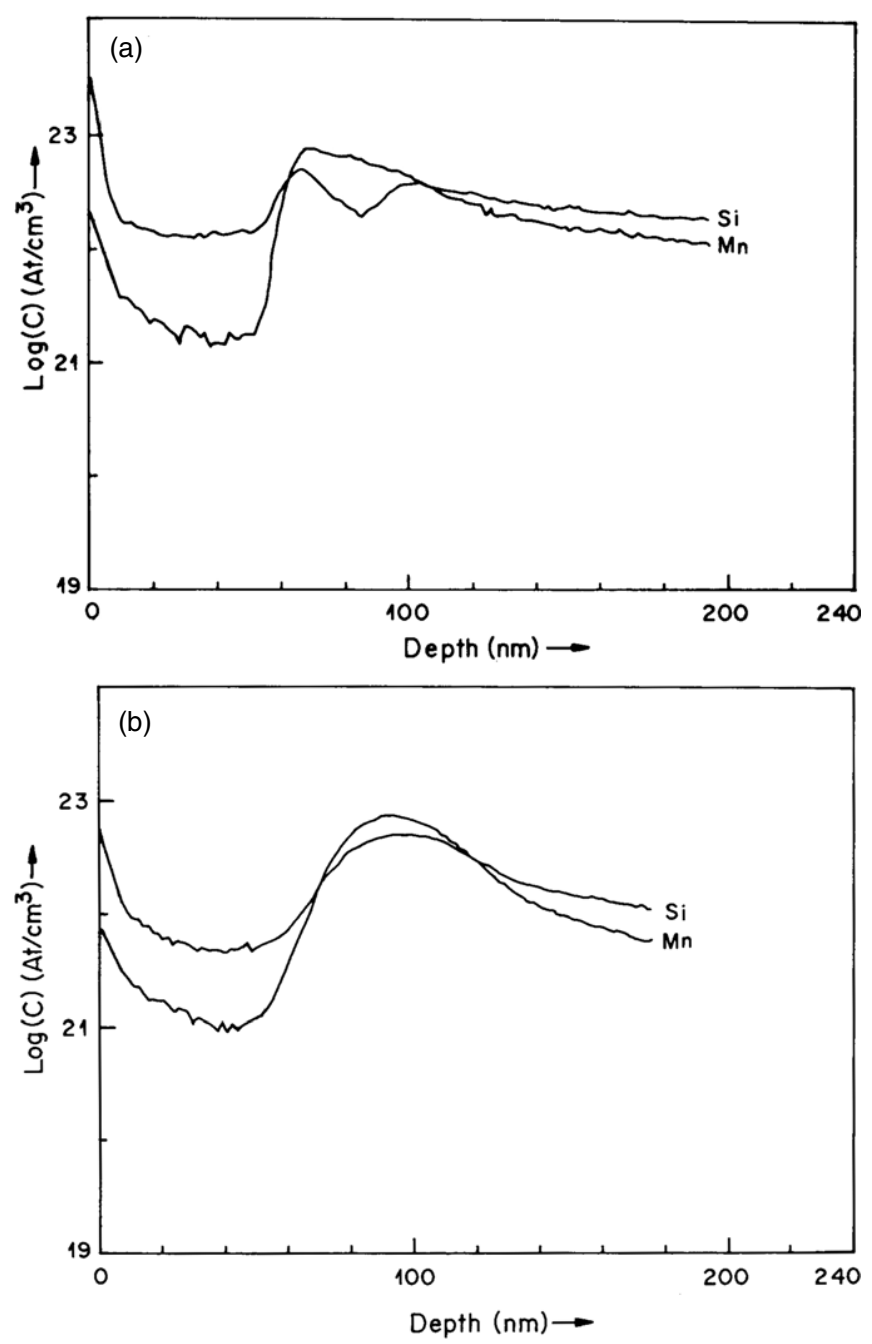

Figure 2. SIMS depth profile of the a-Si/Mn/a-Si interface before irradiation $(a)$ and after irradiation with $(b) 1 \times 10^{14}$ ions $\mathrm{cm}^{-2}$.

to obtain the depth distribution of the elements. The layer thicknesses as determined by this fit are of $\sim 45 \mathrm{~nm}$ for the Si cap layer and $\sim 55 \mathrm{~nm}$ for the Mn layer. The silicon layers that have been deposited are amorphous in nature, and this has been checked by glancing angle x-ray diffraction (GAXRD).

Figure 2(a) shows the $\mathrm{Si}$ top layers up to a depth of approximately $50 \mathrm{~nm}$, after which the Mn concentration suddenly increases suggesting the outset of the Mn layer in between the two Si layers. At this, the Si intensity shows a decrease till the end of the Mn layer. The tailing of the Si and Mn signal after $\sim 100 \mathrm{~nm}$ is due to atomic mixing using $9 \mathrm{KeV}$ primary ion bombardments, which is characteristic of any depth profile data of SIMS in deeper depths when high-energy ions are used. Similarly figure $2(b)$ shows the depth profiles of $\mathrm{Si}$ and $\mathrm{Mn}$ in the sample irradiated at the highest fluence of $1 \times 10^{14}$ ions $\mathrm{cm}^{-2}$. The SIMS profiles show significant changes from the pristine to the samples irradiated with the highest fluence. From SIMS data it can be inferred that the overlap of the in-depth profiles of $\mathrm{Si}$ and $\mathrm{Mn}$ at the highest fluence is due to very high mixing. In all these SIMS depth profiles, the $X$-axis has been converted from the time scale to the depth scale by using the average sputtering rate of $\mathrm{Mn}$

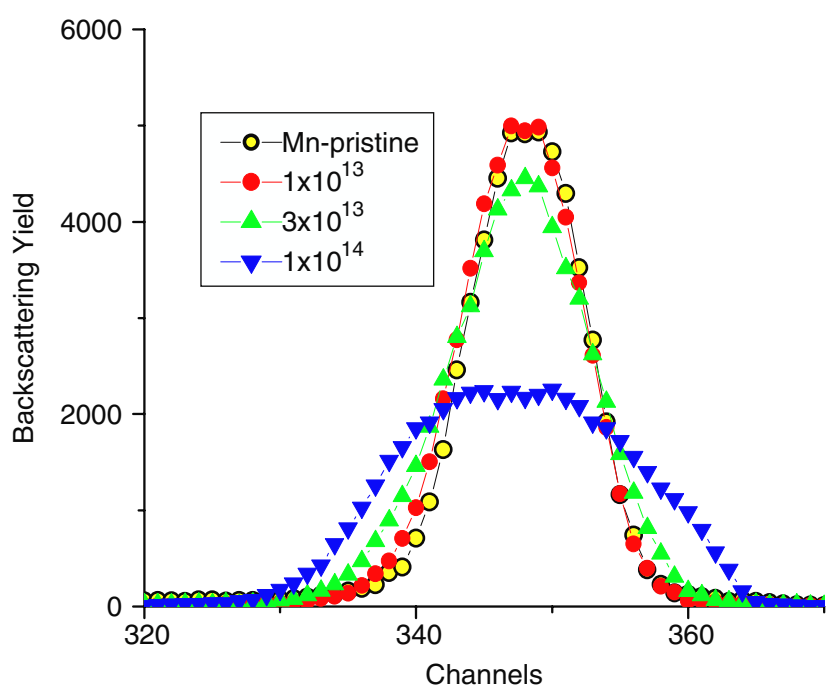

Figure 3. RBS spectra for Mn at all the fluences from $1 \times 10^{13}$ to $1 \times 10^{14}$ ions $\mathrm{cm}^{-2}$.

Table 1. Changes in FWHM values and $\Delta \Omega^{2}$ with the ion fluence for $\mathrm{Si} / \mathrm{Mn} / \mathrm{Si}$.

\begin{tabular}{llll}
\hline $\begin{array}{l}\text { Fluence } \\
(\text { ions cm }\end{array}$ & $\begin{array}{l}\text { FWHM } \\
\text { from } \\
\text { RBS }(\mathrm{nm})\end{array}$ & $\begin{array}{l}\text { Change in } \\
\text { variance } \\
\Delta \Omega_{\text {mix }}^{2}\left(\mathrm{~nm}^{2}\right)\end{array}$ & $k=\Delta \Omega_{\text {mix }}^{2} \Phi$ \\
\hline 0 & 13.9 & - & $\begin{array}{l}\text { The value as estimated } \\
\text { from figure } 5 \text { is } \\
\sim 1000 \mathrm{~nm}^{4}\end{array}$ \\
& & 20.1 & \\
$1 \times 10^{13}$ & 14.6 & 113.1 & \\
$3 \times 10^{13}$ & 17.5 & 942.5 & \\
$1 \times 10^{14}$ & 33.7 & & \\
\hline
\end{tabular}

and Si. The counts $\mathrm{s}^{-1}$ in the $Y$-axis have been normalized to the atomic concentration using the relative sensitivity factor (RSF) from the standard handbook [19].

Figure 3 shows the changes in the part of the RBS spectra related to $\mathrm{Mn}$ atoms at different fluences $\left(1 \times 10^{13}\right.$ $1 \times 10^{14}$ ions $\left.\mathrm{cm}^{-2}\right)$. For the pristine case we observe the highest peak (lowest full width at half maximum (FWHM) value) and as we increase the fluence, the peak height is lowered with an increase in the FWHM value. But a very prominent change is visible at the highest fluence where the peak height has significantly dropped with a change in the slope of the Gaussian, indicating diffusion of $\mathrm{Mn}$ on both sides, i.e. Mn has diffused in both the top and the bottom Si layers. From the values of FWHM [20] we have calculated the change in variance $\Delta \Omega_{\mathrm{RBS}}^{2}$, which is the difference in the change in variance at the fluence $(\Phi)$ and the un-irradiated case $(0)$. By using SRIM [20] we have FWHM $=2.35 \Omega$. Table 1 shows the changes in values of the interface width on irradiation by using RBS.

It should be noted that $\Delta \Omega_{\mathrm{RBS}}^{2}$ is composed of the variance of the interfacial concentration gradient $\Delta \Omega_{\text {mix }}^{2}$ and the fluctuation of the top layer thickness $\Delta \Omega_{\mathrm{d}}^{2}$. The influence of the surface roughness on the RBS spectra is schematically illustrated by Sosa et al [22]. In first order approximation, the total effect at the interface is given by $\Delta \Omega_{\mathrm{RBS}}^{2}=\Delta \Omega_{\mathrm{mix}}^{2}+\Delta \Omega_{\mathrm{d}}^{2}$. 

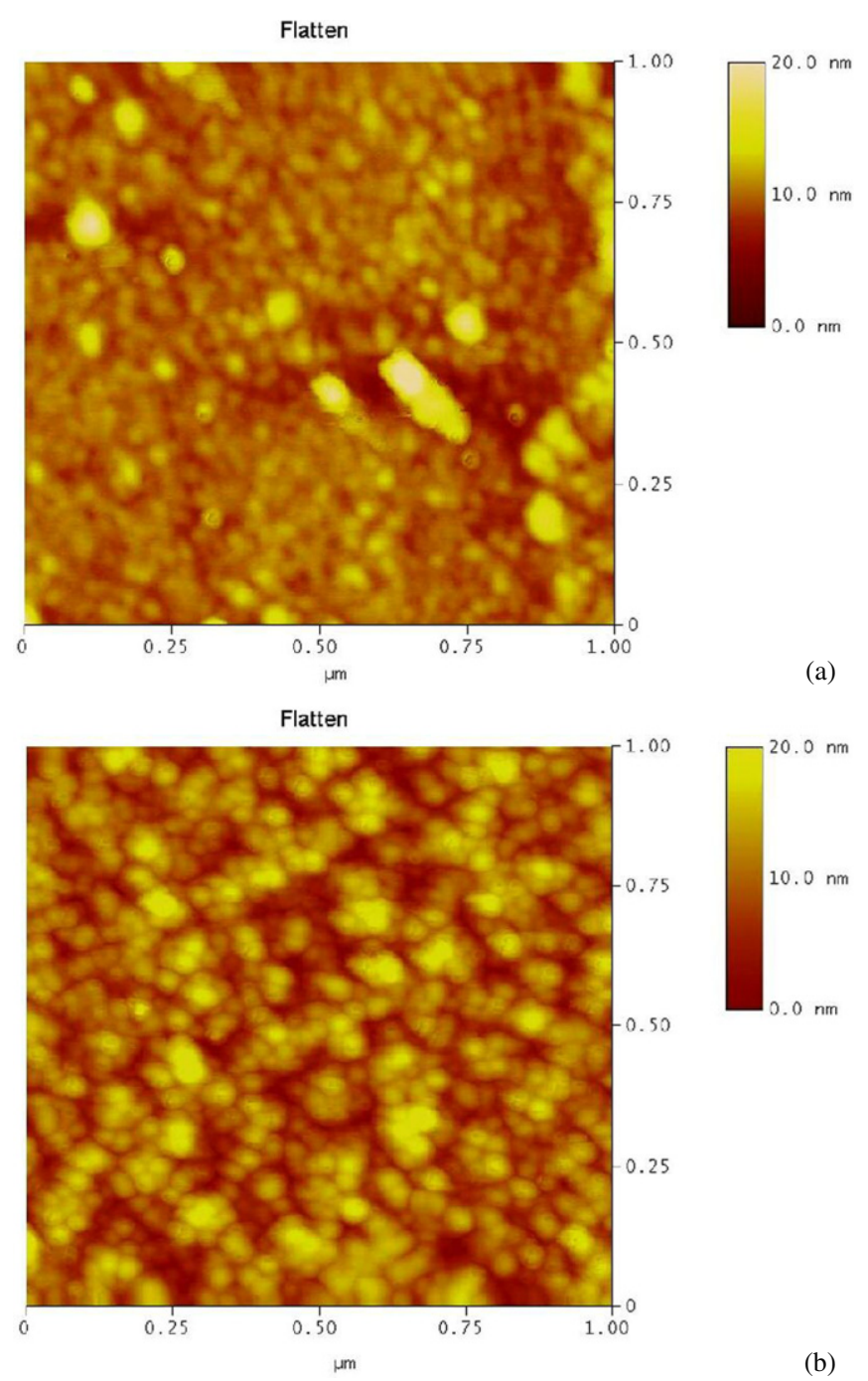

Figure 4. Surface of the pristine $(a)$ and the irradiated $\left(1 \times 10^{14}\right.$ ions $\left.\mathrm{cm}^{-2}\right)$ case $(b)$.

If the contribution from the surface is negligible then we can affirmatively conclude that $\Delta \Omega_{\mathrm{RBS}}^{2} \cong \Delta \Omega_{\mathrm{mix}}^{2}$. From the AFM micrographs (figure 4 ) it is calculated that the change in roughness values is insignificant and can be ignored. $\Omega_{\mathrm{d}}$ hardly changes with the variation in fluence from pristine to $1 \times 10^{14}$ ions $\mathrm{cm}^{-2}$. In our system it has been found that the contribution from the surface roughness is negligible and thus the change in variance is basically due to interface mixing.

A linear increase of $\Delta \Omega_{\text {RBS }}^{2}$ with the ion fluence $\Phi$ is seen in figure 5. The mixing rate $\left(k=\Delta \Omega_{\mathrm{RBS}}^{2} / \Phi\right)$ as obtained from the slope of the line is $\sim 1000 \mathrm{~nm}^{4}$. This value for the mixing rate is quite high in comparison with other systems that we have studied $[10,12]$.

Since the present case involves $\mathrm{Mn}$, a metal, the applicability of the Coulomb explosion model is ruled out because of the high mobility of conduction electrons. The other possibility is the thermal spike model, which has already been effectively used even in several insulator/insulator systems [23] also to show that interface mixing is due to inter-diffusion in the melt phase. Consequently, to verify the hypothesis of melt phase diffusion across the interface using the thermal spike

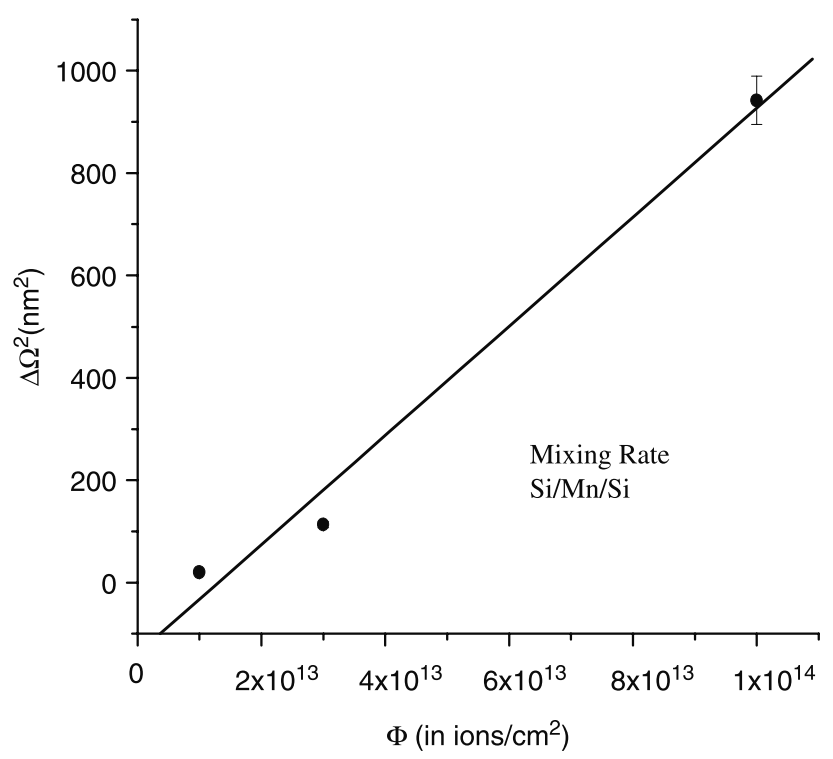

Figure 5. Variation of $\Delta \Omega$, with the ion fluence $\Phi$.

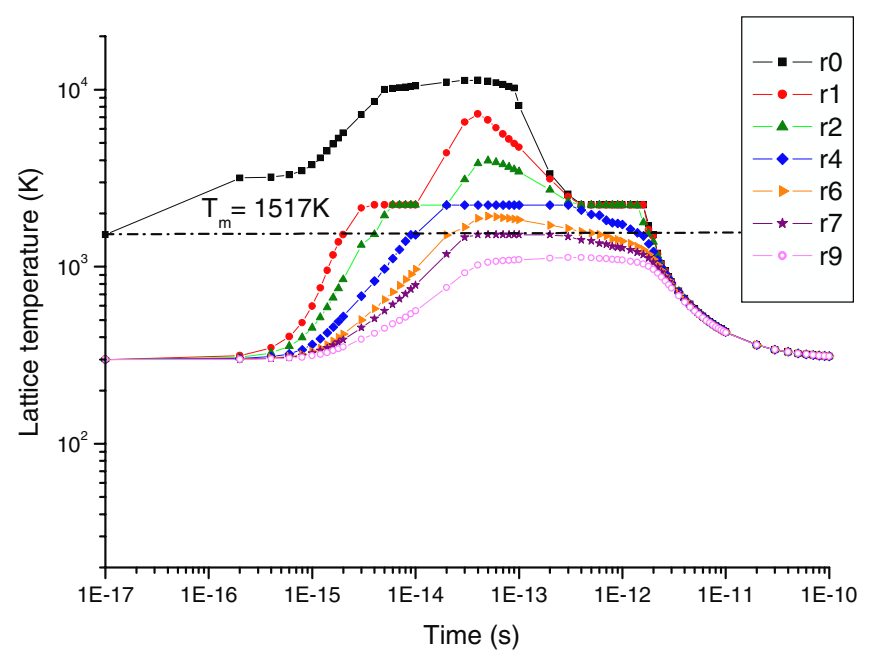

Figure 6. Lattice temperature of Mn with time for various radii $r$ (in $\mathrm{nm}$ ) of cylinders around $120 \mathrm{MeV} \mathrm{Au}$ ion path as calculated using the thermal spike model.

model the diffusivity at the interface has been estimated for this system. For examining this aspect, the diffusion coefficient across the interface [11] for $\mathrm{Mn}$ is given as $D=\Delta \Omega_{\mathrm{RBS}}^{2} 2 t_{\mathrm{n}}$, where $t_{\mathrm{n}}=$ diffusion time. The diffusion time during the transient melt phase is obtained as $t_{\mathrm{n}}=\left(\Phi / \Phi_{\mathrm{c}}\right) \times t_{\mathrm{S}}$, where $t_{\mathrm{S}}=$ the duration of the melt phase of $\mathrm{Mn}, \Phi=$ the maximum fluence and $\Phi_{\mathrm{c}}=1 /(2 r)^{2}$ is the fluence for the complete overlap of the ion tracks and $r$ is the track radius. The evolution of the Mn-lattice temperature with time for a number of cylinders of different radii around the path for $120 \mathrm{MeV}$ $\mathrm{Au}$ ions at normal incidence is calculated according to the thermal spike model which is shown in figure 6 . The melting temperature $\left(T_{\mathrm{m}}\right)$ of $\mathrm{Mn}$ is $1517 \mathrm{~K}$; thus up to a $7 \mathrm{~nm}$ cylinder around the ion path remains at or above the meting temperature for about $0.16 \mathrm{ps}$. These values in $\mathrm{Mn}$ are the track radius ' $r$ ' and the spike time ' $t_{\mathrm{S}}$ '. Thus, the track diameter $2 r=7 \mathrm{~nm}$ and $t_{\mathrm{S}}=0.16 \mathrm{ps}$, respectively. Using the values of $r, t_{\mathrm{S}}$ and 
experimental values of $\Delta \Omega_{\mathrm{RBS}}^{2}, \Phi_{\mathrm{c}}$ and $\Phi$, the diffusivity $D$ at the interface turns out to be of the order of $\sim 10^{-6} \mathrm{~m}^{2} \mathrm{~s}^{-1}$, which is the characteristic of the liquid phase, thus proving the IBM in the framework of the thermal spike model $[5,8]$.

Mixing can be attributed to inter-diffusion of $\mathrm{Si}$ and Mn atoms across the interface during the transient melt phase according to the thermal spike model. Both these elements are sensitive to $S_{\mathrm{e}}$, and in the case of $\mathrm{Si}$, the calculated value for $S_{\mathrm{e}}\left(\sim 14 \mathrm{keV} \mathrm{nm}^{-1}\right)$ is much above the $S_{\mathrm{e}}$ threshold $\left(\sim 9 \mathrm{keV} \mathrm{nm}^{-1}\right)$ [24]. In the case of $\mathrm{Mn}$, the $S_{\mathrm{e}}$-threshold value is not available, but since the mixing rate in this case is very high it can be predicted that the $S_{\mathrm{e}}$ threshold in Mn must be surpassed.

In the case of $\mathrm{Mn}$, the highest mixing rate with respect to that of $\mathrm{V}, \mathrm{Fe}, \mathrm{Co}[10,12]$ is due to the high value for the electron-phonon [e-p] coupling factor ' $g$ ' [8] which is an important parameter contributing to mixing behaviour. Thus, the dependence of mixing on the e-p coupling factor further indicates that the thermal spike model is suitable for explaining this interface-mixing phenomenon.

\section{Conclusion}

This work reports the SHI-induced interface mixing in a-Si/Mn/a-Si thin film system when irradiated by $120 \mathrm{MeV} \mathrm{Au}$ ions, due to the dominance of electronic energy loss. Mixing has been quantified in terms of the mixing rate for the first time in the $\mathrm{Si} / \mathrm{Mn} / \mathrm{Si}$ system. Finally, it can be inferred that the cause of mixing in this system is due to diffusion across the interface during a transient molten stage, under the framework of the thermal spike model.

\section{Acknowledgments}

One of the authors (Diva) is thankful to the Council of Scientific and Industrial Research, New Delhi, for the financial support to carry out this work. We are also thankful to Dr Ram Kishore of NPL, Delhi for his constructive suggestions and Ms Neeti Tripathi for the AFM data.

\section{References}

[1] Bolse W 2003 Radiat. Meas. 36597

[2] Dufour C, Bauer Ph, Marchal G, Grilhe J, Jaouen C, Pacaua J and Jousset J C 1993 Eur. Phys. Lett. 21671

[3] Srivastava S K, Avasthi D K, Assmann W, Wang Z G, Kucal H, Jacquet E, Carstanjen H D and Toulemonde M 2005 Phys. Rev. B 71193405

[4] Assmann W, Dobler M, Avasthi D K, Kruijer S, Mieskes H D and Nolte H 1998 Nucl. Instrum. Methods B 146271

[5] Avasthi D K, Assmann W, Nolte H, Mieskes H D, Ghosh S and Mishra N C 2000 Nucl. Instrum. Methods B 1661345

[6] Bolse W and Schattat B 2002 Nucl. Instrum. Methods B 190173

[7] Lesueur D and Dunlop A 1993 Radiat. Eff. Defects Solids 126123

[8] Wang Z G, Dufour C, Penmier E and Toulemonde M 1994 J. Phys.: Condens. Matter 66733

[9] Schattat B, Bolse W, Klaumunzer S, Harbsmeier F and Jasenek A 2003 Appl. Phys. A 76165

[10] Wang E, Angert N, Trantmann C and Vetter J 1995 J. Adhes. Sci. Technol. 91523

[11] Diva K, Kabiraj D, Chakraborty B R, Shivaprasad S M and Avasthi D K 2004 Nucl. Instrum. Methods B 222169

[12] Kumar S, Chauhan R S, Khan S A, Bolse W and Avasthi D K 2006 Nucl. Instrum. Methods B 244194

[13] Chakraborty B R, Kabiraj D, Diva K, Pivin J C and Avasthi D K 2006 Nucl. Instrum. Methods B 244209

[14] Chakraborty B R, Halder S K, Karar N, Kabiraj D and Avasthi D K 2005 J. Phys. D: Appl. Phys. 382836

[15] Murarka S P 1983 Silicides for VLSI Applications (New York: Academic)

[16] Higai S and Ohno T 2002 Phys. Rev. B65 165309

[17] Mayer J W, Tsaur B Y, Lau S S and Hung L S 1981 Nucl. Instrum Methods Phys. Res. B 182/183 1

[18] Pivin J C, Dimova-Malinovska D, Sendova-Vassileva M and Nikolaeva M 2001 Nucl. Instrum. Methods B 174453

[19] Chu W K, Mayer J W and Nicolet M-A 1978 Backscattering Spectrometry (New York: Academic)

[20] Wilson R G, Stevie F A and Magee C W Secondary Ion Mass Spectrometry (New York: Wiley) p 2.1-1

[21] Ziegler J F, Biersac J P and Littmark U 1985 The Stopping and Range of Ions in Matter (New York: Pergamon)

[22] Sosa A C, Schaaf P, Bolse W, Lieb K P, Gimbel M, Geyer U and Tosello C 1996 Phys. Rev. B 5314795

[23] Schattat B, Bolse W, Klaumunzer S, Harbsmeier F and Jasenek A 2003 Appl. Phys. A 76165

[24] Dhuri P, Gupta A, Chaudary S M., Phase D M and Avasthi D K 1999 Nucl. Instrum. Methods B 156148 